Abstract 103(P) Table 1 Percentage of staff that gave an agree/strongly agree response to each survey statement

\begin{tabular}{|c|c|c|c|c|c|c|c|c|c|c|c|}
\hline & 1 & 2 & 3 & 4 & 5 & 6 & 7 & 8 & 9 & 10 & 11 \\
\hline Pre & $66.67 \%$ & $86.67 \%$ & $93.33 \%$ & $80 \%$ & $60 \%$ & $40 \%$ & $80 \%$ & $66.667 \%$ & $60 \%$ & $73.33 \%$ & $86.67 \%$ \\
\hline Post & $80 \%$ & $90 \%$ & $60 \%$ & $100 \%$ & $80 \%$ & $50 \%$ & $90 \%$ & $100 \%$ & $90 \%$ & $80 \%$ & $80 \%$ \\
\hline
\end{tabular}

9. Teaching opportunities for the day are highlighted

10. When I am the one giving handover, I feel comfortable doing so

11. Paediatric handovers at Peterborough are enjoyable and non-intimidating.

Percentage of staff that gave an agree/strongly agree response to each survey statement (table 1).

Conclusion The intervention improved the quality of our morning handover sessions. A follow up survey is being put in place to assess whether these improvements have been sustained.

\section{G104(P) CRIT: CHILDREN RECEIVING IMMUNOSUPPRESSIVE THERAPY: A CROSS-SPECIALITY REVIEW OF PRACTICE AT A TERTIARY CHILDREN'S HOSPITAL}

${ }^{1} \mathrm{C}$ Tsilifis, ${ }^{1} \mathrm{~A}$ Battersby, ${ }^{2} \mathrm{E}$ Williams, ${ }^{2} \mathrm{~T}$ Flood, ${ }^{2} \mathrm{~A}$ Flinn, ${ }^{3} \mathrm{~F}$ McErlane, ${ }^{3} \mathrm{~S}$ Sampath. ${ }^{1}$ General Paediatrics, Great North Children's Hospital, Newcastle upon Tyne, UK; ${ }^{2}$ Department of Paediatric Immunology, Great North Children's Hospital, Newcastle upon Tyne, UK; ${ }^{3}$ Department of Paediatric Rheumatology, Great North Children's Hospital, Newcastle upon Tyne, UK

\subsection{6/archdischild-2020-rcpch.82}

Aims Immunosuppression has become integral to the management of a wide range of childhood illness. Although total numbers of children and young people (CYP) on immunosuppression is unknown, they are thought to be increasing. Multiple paediatric sub-specialities initiate and monitor different immunosuppressive therapies with anecdotal variation in prescribing and monitoring practices. To understand existing variations in care, we undertook a retrospective review, focussing on:

- Number of CYP on immunosuppression;

- Drug monitoring and antimicrobial prophylaxis;

- Numbers of serious infections.

Methods CYP attending our hospital on immunosuppressive medication were identified from speciality teams (excluding immunology/oncology patients). Immunosuppressive agents were defined as:

- Long-term/high-dose steroids

- Cytotoxic drugs

- Biologic agents/monoclonal antibodies

Data were collected on a representative sample of patients under each speciality:

- Diagnosis;

- Current/past immunosuppression;

- Current/past antimicrobial prophylaxis;

- Consideration of risk for specific infections;

- Serious infective episodes.

Existing speciality/departmental guidance on prescribing, monitoring and surveillance was collected.
Results 416 CYP on immunosuppression identified; data were collected on 77 (18.5\%), with cross-speciality representation.

$47 / 77$ children are currently prescribed $\geq 2$ agents with $8 /$ 77 presently off immunosuppression. $46 / 77$ were not prescribed prophylaxis at any point. In those who were, cotrimoxazole $(n=28)$ was commonest.

$54 / 77$ patients had past VZV exposure documented or tested. $7 / 77$ patients attended after chickenpox exposure; 2 required admission for treatment. 10/77 patients were hospitalised for possible bacterial infection; none had proven bacteraemia but 2 developed cryptosporidiosis.

All patients had FBC checked with varying frequency (weekly-once only). 14/77 developed lymphopaenia; of these, 8 had subsets checked. There was no clear relationship between speciality and monitoring frequency.

Two specialities were able to provide departmental guidance for the management of intercurrent infection.

Conclusions There is a large population of CYP on a wide range of immunosuppression. There is inter-speciality variation in the agents prescribed, monitoring schedules, infection risk stratification and antimicrobial prophylaxis. Departmental protocols are uncommon and not readily accessible outside of speciality or hospital, making out-of-hours decisions unnecessarily challenging.

This wide variation in practice and lack of evidence-based guidance is unacceptable.

Regional immunosuppression guidance may improve the quality of care offered to immunosuppressed children in our region.

\section{G105(P) INITIATIVE TO IMPROVE CLINICAL CODING FOR PATIENT ADMISSION EPISODE (INPATIENT) BY RECORDING PATIENT CO-MORBIDITIES}

${ }^{1} \mathrm{M}$ Joshi, ${ }^{1} \mathrm{~S}$ Bandi, ${ }^{2} \mathrm{R}$ Ball. ${ }^{1}$ Department of Paediatrics, Leicester Royal Infirmary, University Hospitals of Leicester NHS Trust, Leicester, UK; ${ }^{2}$ Transformation Lead, Womens and Childrens CMG, Management, University Hospitals of Leicester NHS Trust, Leicester, UK

\subsection{6/archdischild-2020-rcpch.83}

Background We reviewed benchmarking data about comorbidities in paediatric medicine provided by Civil Eye Research compared to other tertiary hospital. The data showed that our hospital (paediatric medicine) was on the lowest spectrum compared to other hospitals of similar size. This prompted us to look it in depth with an aim to improve our recording of co-morbidities and clinical coding.

Aim To record patient co-morbidities during each admission episode.

Methods We applied Quality Improvement (QI) methodology (PDSA cycle- Plan, Do, Study, Act) in this project.

Coding champion's role helped to bridge the gap between clinical coding and the medical teams. We identified relevant co-morbidities and designed a paediatric co-morbidity sheet. These sheets were formally launched on all the paediatric 
wards and medical \& nursing staff was updated via regular teaching and educational sessions.

We completed one audit cycle by retrospectively reviewing 20 patient medical notes each time (May-June 2019 \&amp August-September 2019).

Results Our first audit showed that only 20\% (4/20) patient notes had a co-morbidity sheet whereas the re audit post educational sessions helped to improve it to $75 \%(15 / 20)$.

Since implementing the co-morbidity sheet and using it regularly from April 2019, our low bracket coding (between 0-1 co morbidity) reduced from $40 \%$ to $27 \%$; mid bracket coding (2-5 comorbidities) improved from $47 \%$ to $54 \%$ and the highest bracket coding $(6+$ co morbidities) improved from $13 \%$ to $19 \%$. Overall this resulted in a financial gain of $£ 195,000$ since the implementation of co-morbidity sheet.

Conclusion Comorbidities are conditions that are present in association with a current illness with which a patient is admitted. It is very important to document comorbidities in clinical notes and discharge summaries which can then be coded accurately. This in turn helps to secure appropriate funding to provide appropriate and effective patient care. Our experience shows that using a comorbidity sheet based on common health conditions helps clinical as well as coding staff to improve clinical coding. Regular updates via educational sessions about the benchmarking data and PDSA cycles are key to sustain this improvement.

\section{G106(P) ABSTRACT WITHDRAWN}

\section{G107(P) REDUCING PARACETAMOL MEDICATION ERRORS IN CHILDREN}

1J Crook, ${ }^{2} \mathrm{H}$ Yorke, ${ }^{2} \mathrm{R}$ Cooper. ${ }^{1}$ Pharmacy, Chelsea and Westminster FT, London, UK;

${ }^{2}$ Paediatric Department, Chelsea and Westminster FT, London, UK

10.1136/archdischild-2020-rcpch.84

Introduction Paracetamol is one of the most common medicines administered to children nationally. Accidental overdoses with intravenous (IV) paracetamol in children $<10 \mathrm{~kg}$ have been reported, including fatalities. High numbers of medication incidents (MI) involving prescribing and administration of paracetamol on paediatric inpatient wards were reported. To reduce the level of harm the multidisciplinary team reviewed incident trends and implemented selected interventions.

Method MI were recorded and reviewed retrospectively every quarter (Q) from Q1 March-May 2018 to Q2 July-Sept 2019. Ethics approval was not required for this study. Selected measures were then used to track changes in incident numbers and trends on implementation of intervention.

Measures

- Number of paracetamol incidents

- Level of harm

- Incident type - categorised into trends.

Intervention 1 (Q3 October-December 2018) Paracetamol medication safety bulletin, highlighting risks associated prescribing paracetamol in children was circulated to staff. Standardised dosing approved in cross-site guideline. Targeted staff education around incident data, trends and top tips. Removal of multi-route screens on the electronic prescribing system to reduce number of IV paracetamol prescriptions.

Intervention 2 (Q1 March-May 2019) Introduction of dose/ volume checks for intravenous paracetamol included in the paediatric IV guide.

Results

Intervention 1

- $75 \%$ reduction in number of paracetamol errors. A $65 \%$ reduction in the total percentage MI involving paracetamol.

- $50 \%$ reduction in number of MI with a reported level of harm, the level of harm remained the same

- $100 \%$ reduction of oral dosing errors. $50 \%$ reduction in number of IV dosing errors for babies $<10 \mathrm{~kg}$ and $40 \%$ reduction in duplication of doses.

Intervention 2

No reported IV paracetamol dosing errors for babies $<10$ $\mathrm{kg}$ in Q1-2 2019.

Conclusion A significant level of harm has been reported with paracetamol MI in children. Regular review of MI numbers and trends, with targeted education and standardisation of practice can reduce number of $\mathrm{MI}$, including those causing harm. Increased risk of adverse effects of paracetamol overdose in children means the level of harm was not reduced. Future work is needed to reduce incident numbers involving dose duplication.

\section{G108(P) IMPLEMENTATION OF A PROLONGED JAUNDICE PATHWAY: A QUALITY IMPROVEMENT INITIATIVE. TIME FOR NICE TO HAVE A RETHINK!}

SK Kumar, PS Surana. Neonatal Unit, University Hospitals Birmingham NHS Trust, Birmingham, UK

\subsection{6/archdischild-2020-rcpch.85}

Introduction Prolonged neonatal jaundice (PNJ) is mostly benign and self-limiting; however, it could represent a serious pathology such as biliary atresia; timely identification of which is vital. NICE guidance is available on PNJ, however, hospital referrals of all such infants can lead to over-investigation, unnecessary pressure on hospital resources and undue parental anxiety. Hence, there needs to be a fine balance between over-investigating well infants and timely detection of serious pathology.

Background Previously, all cases of community diagnosed PNJ were referred to consultant clinic in our tertiary neonatal unit. An audit in 2013(Jan-Dec) revealed a high number (222) of such referrals. Of these, $38 \%$ were not even clinically jaundiced at the time of clinic attendance. The rest had unnecessary investigations-91\% and $61 \%$ had liver and thyroid function tests respectively; not recommended by NICE.

Aim The above audit prompted a quality improvement initiative of implementing an alternative pathway to streamline PNJ referrals in Sep'15. As per the pathway, community midwives perform direct and indirect bilirubin on infants and refer only if any of the following criteria are met- not thriving, pale stools, total bilirubin $>200$ ummol/L, direct bilirubin $>20$ ummol/L or worsening jaundice despite blood test.

Method A re-audit was performed in May'19 to evaluate the efficacy of the pathway over 3 years(Mar'16-Feb'19) and to assess any delay in diagnosing serious pathology.

Results Table 1 compares results before and after implementation of PNJ pathway. 